\title{
PROPRIEDADES FÍSICO-MECÂNICAS DE PAINÉIS LVL PRODUZIDOS COM TRÊS ESPÉCIES AMAZÔNICAS
}

\author{
Nerci Nina Lima ${ }^{1}$, Lourival Marin Mendes $^{2}$, Vânia Aparecida de Sá2*, Lina Bufalino² \\ *Autora para correspondência: vania_desa@yahoo.com.br
}

RESUMO: Neste trabalho, objetivou-se avaliar o desempenho físico-mecânico de painéis de lâminas paralelas (LVL) produzidos com diferentes composições de espécies tropicais amazônicas e Pinus oocarpa. Foram utilizadas três espécies tropicais: Cordia goeldiana Huber. (Freijó), Parkia gigantocarpa Ducke (Faveira) e Brosimum parinarioides Ducke (Amapá doce). Para comparação, foi utilizada madeira de Pinus oocarpa proveniente de plantio experimental. Três tipos de combinações de lâminas foram avaliados: (i) uma espécie, (ii) combinação das três espécies amazônicas e (iii) combinação de espécies amazônicas com Pinus oocarpa. Avaliouse também a velocidade de propagação acústica com o stress wave timer para a determinação do módulo de elasticidade dinâmico (MOEd), que foi correlacionado com o módulo de elasticidade estático (MOEe) do painel. Os painéis produzidos com espécies da Amazônia apresentaram menor absorção de água total em relação aos painéis de Pinus oocarpa. Para as propriedades mecânicas, a menor resistência foi verificada para painéis de faveira. Não houve diferença estatística entre os painéis para a resistência ao cisalhamento na linha de cola. O stress wave timer poderia ser utilizado para predição do MOEe a partir do MOEd.

Palavras-chave: Painel estrutural, stress wave timer, madeiras tropicais.

\section{MECHANICAL AND PHYSICAL PROPERTIES OF LVL PANELS MADE FROM THREE AMAZONIC SPECIES}

\begin{abstract}
This work aimed to evaluate the physical-mechanical performance of laminated veneer lumber (LV) produced with different compositions of tropical Amazonian species and Pinus oocarpa. Three tropical species were used: Cordia goeldiana Huber. (Freijó), Parkia gigantocarpa Ducke (Faveira) and Brosimum parinarioides Ducke (Amapá doce). For comparison, Pinus oocarpa wood obtained from an experimental stand was used. Three types of veneer combination were evaluated: (i) one species; (ii) the three Amazonian species combination and (iii) combination of Amazonian species with Pinus oocarpa. The acoustic propagation velocity was also evaluated with the stress wave timer for correlation between dynamic modulus of elasticity (MOEd) and static modulus of elasticity (MOEs) of the panel. Panels produced with Amazonian species presented lower water absorption than Pinus oocarpa panels. Regarding the mechanical properties, the lowest strength was verified for "faveira" panels. There was no statistical difference among panels in glue line shear strength. The stress wave timer could be used for prediction of MOEs by using MOEd.
\end{abstract}

Key words: Structural panel, stress wave timer, tropical woods.

\section{INTRODUÇÃO}

Antes do desenvolvimento da indústria de painéis reconstituídos, a aplicação de produtos à base de madeira na construção civil estava limitada às madeiras serradas, as quais, geralmente, não oferecem a estabilidade dimensional e uniformidade adequadas para materiais estruturais (COLAK et al., 2007; ÖZÇIFÇI, 2007). O uso de estruturas de madeira já é comum nos Estados Unidos e Canadá. O LVL é um dos compostos estruturais mais utilizados. No entanto, no Brasil, não são produzidos comercialmente (MCKEEVER, 1997).

A maioria dos trabalhos científicos nacionais sobre painéis LVL foi realizada com espécies dos gêneros Pinus (BORTOLETTO JÚNIOR, 2009; GABRIEL, 2007)

${ }^{1}$ Universidade Federal do Amazonas - Manaus, Amazonas, Brasil

${ }^{2}$ Universidade Federal de Lavras - Lavras, Minas Gerais, Brasil e Eucalyptus (CARVALHO et al., 2004; IWAKIRI et al., 2008). No entanto, os estudos visando a verificar o potencial de lâminas de madeiras tropicais para a produção de painéis LVL são escassos (IWAKIRI et al., 2010).

Aliado à demanda atual por produtos à base de madeira, é necessário considerar que a produção madeireira na Amazônia não é condizente com toda a sua potencialidade. Isso ocorre porque o sistema de produção regional ainda é centrado no corte seletivo de espécies, ocasionando com isso um gradual empobrecimento da floresta e talvez a extinção das espécies mais intensamente exploradas. Nesse processo, diversas espécies madeireiras não têm seu potencial estudado e deixam de ganhar espaço no mercado nacional e internacional (ARAÚJO, 2002; BARBOSA et al., 2001).

Cerne, Lavras, v. 19, n. 3, p. 407-413, jul./set. 2013 
Teoricamente, qualquer espécie ou combinação de espécies pode ser empregada na produção de painéis reconstituídos de madeira como o LVL, desde que as propriedades físicas e mecânicas resultantes estejam de acordo com as normas de comercialização (MOODY; HERNANDEZ, 1997).

Uma maneira para se aproveitar espécies madeireiras de baixa densidade básica é posicionando suas lâminas na camada mais interna do painel, mas mantendose as de maior densidade básica na superfície, com o intuito de não prejudicar a qualidade final do produto. Esse princípio é baseado no fato de que as lâminas da capa são as regiões que sofrem maior estresse quando os painéis são submetidos a esforços mecânicos, especialmente durante o teste de flexão estática (DING et al., 1995; H'NG et al., 2010; MOHAMAD et al., 2011).

Dessa forma, produtos engenheirados de madeira como o LVL ampliam as alternativas de utilização dos recursos florestais existentes, pois lâminas de madeira de qualidades distintas podem ser aproveitadas pelo seu posicionamento adequado na estrutura do painel de forma a não prejudicar as propriedades tecnológicas do produto (DING et al., 1995).

Outra vantagem relevante da combinação de espécies é a possibilidade de se utilizar madeiras de maior disponibilidade e menor custo, que não poderiam ser utilizadas isoladamente para a produção de LVL's estruturais (BURDURLU et al., 2007). Para o Brasil, isso pode ser especialmente relevante no que se refere à utilização de lâminas de madeiras da Amazônia de boa qualidade e alto custo na capa dos painéis, mantendo-se lâminas de espécies oriundas de plantio florestal, como as diversas espécies dos gêneros Pinus e Eucalyptus, no interior de painéis.

Neste trabalho, objetivou-se avaliar o desempenho físico-mecânico de painéis de lâminas paralelas (LVL) produzidos com diferentes composições de espécies tropicais da Amazônia e Pinus oocarpa.

\section{MATERIAL E MÉTODOS}

\subsection{Obtenção e preparo da matéria-prima}

Foram utilizadas três espécies tropicais da Amazônia: Freijó (Cordia goeldiana Huber), Faveira (Parkia gigantocarpa Ducke) e Amapá doce (Brosimum parinarioides Ducke). As madeiras são procedentes da área de manejo florestal da Fazenda Rio-Capim pertencente à empresa Cikel Brasil Verde S.A, localizada no município de Paragominas - PA. Foram coletadas e abatidas três árvores de cada espécie. De cada árvore, foi retirada uma tora da base com comprimento de $1,3 \mathrm{~m}$.

A madeira de Pinus oocarpa foi utilizada como parâmetro de comparação. Uma árvore foi coletada no Campus da Universidade Federal de Lavras (UFLA), Lavras - MG, da qual foram obtidas duas toras com comprimento de $0,60 \mathrm{~m}$. Na Tabela 1, estão apresentadas as características das espécies utilizadas.

Tabela 1 - Descrição das espécies.

Table 1 -Description of the species.

\begin{tabular}{lccc}
\hline Espécie & $\mathrm{DM}(\mathrm{cm})$ & $\mathrm{HC}(\mathrm{m})$ & $\mathrm{DB}\left(\mathrm{g} / \mathrm{cm}^{3}\right)$ \\
\hline Freijó & 0,58 & 28,68 & 0,504 \\
Faveira & 1,11 & 18.93 & 0,281 \\
Amapá doce & 0,70 & 19,09 & 0,417 \\
Pinus & 047 & 21,00 & 0,460 \\
\hline
\end{tabular}

DM: diâmetro médio a $1,30 \mathrm{~m}$ do solo; HC: altura comercial; DB: densidade básica.

\subsection{Processo de laminação}

As espécies amazônicas foram laminadas na própria empresa. As toras foram previamente aquecidas em tanques a $96{ }^{\circ} \mathrm{C}$, durante 24 horas pelo processo de cozimento a vapor, com exceção da madeira de faveira que foi laminada sem aquecimento em função de sua baixa densidade. Após laminação, as lâminas foram guilhotinadas nas dimensões finais de $110 \mathrm{~mm}$ x $120 \mathrm{~mm}$ para transporte até a Universidade Federal de Lavras.

As toras de Pinus oocarpa foram laminadas após cozimento em tanques com água a $70^{\circ} \mathrm{C}$, durante 24 horas em laboratório.

Ambos os tornos laminadores foram ajustados para a obtenção de lâminas com espessura nominal de $2 \mathrm{~mm}$. As lâminas foram guilhotinadas para as dimensões nominais de $500 \mathrm{~mm}$ x $500 \mathrm{~mm}$ e secas ao ar livre até $12 \%$ de umidade de equilíbrio da madeira.

\subsection{Manufatura dos painéis LVL}

A classificação das lâminas foi realizada em função da qualidade quanto à ausência de nós, aspereza de superfície, ausência de fungos e defeitos de secagem. As melhores lâminas foram posicionadas na capa dos painéis. As dimensões pré-definidas para os painéis foram de $500 \mathrm{~mm}$ x $500 \mathrm{~mm}$ x $18 \mathrm{~mm}$ com 9 lâminas cada. Na Tabela 2, são apresentadas as composições dos tratamentos.

Cerne, Lavras, v. 19, n. 3, p. 407-413, jul./set. 2013 
Tabela 2 - Composição dos painéis.

Table 2 - Composition of panels.

\begin{tabular}{cc}
\hline Tratamento & Composição \\
\hline $\mathrm{Fr}$ & Lâminas de Freijó \\
$\mathrm{Ap}$ & Lâminas de Amapá \\
$\mathrm{Fa}$ & Lâminas de Faveira \\
$\mathrm{Pi}$ & Lâminas de Pinus \\
$\mathrm{M}_{1}$ & Mistura das espécies da Amazônia \\
$\mathrm{M}_{2}$ & Mistura com inclusão de Pinus \\
\hline
\end{tabular}

O parâmetro utilizado para a disposição das lâminas dos painéis mistos foi a densidade básica da madeira. $\mathrm{Na}$ mistura 1, composta por espécies da Amazônia, o freijó, de maior densidade básica, foi posicionado nas faces dos painéis, o amapá na camada de transição, e a faveira na camada interna. Para a mistura 2, o pinus comercial foi incluído na camada mais interna, sendo a faveira de menor densidade básica, posicionada entre as lâminas dessa espécie, conforme Figura 1.

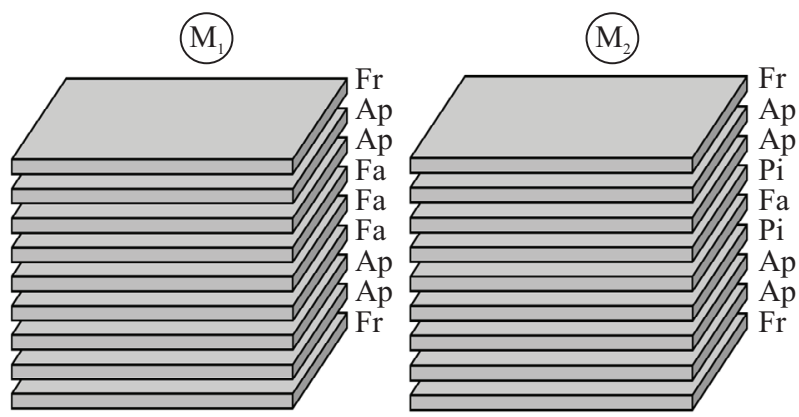

Figura 1 - Esquema de posicionamento das lâminas nos painéis dos tratamentos $\mathrm{M}_{1}$ e $\mathrm{M}_{2}$.

Figure 1 - Scheme of veneer positioning in panels of M1 and M2 treatments.

Os esquemas de posicionamento das lâminas utilizados para as misturas estão apresentados na Figura 1.

Foi utilizado o adesivo resorcinol-formaldeídeo na gramatura de $160 \mathrm{~g} / \mathrm{m}^{2} \mathrm{em}$ linha simples. A aplicação do adesivo foi manual com auxílio de uma espátula para espalhamento.

Os painéis foram prensados em temperatura ambiente com pressão de $12 \mathrm{kgf} / \mathrm{cm}^{2}$, durante 7 horas. Após a confecção dos painéis, os mesmos foram climatizados a uma umidade de $65 \pm 3 \%$ e temperatura de $20 \pm 1{ }^{\circ} \mathrm{C}$.

\subsection{Confecção e ensaio dos corpos-de-prova}

A confecção dos corpos de prova e procedimentos dos ensaios foram realizados segundo as recomendações da norma NBR 31:000.05-001 (ASSOCIAÇÃO BRASILEIRA DE NORMAS TÉCNICAS - ABNT, 2004), para compensados, adaptada. Em razão das dimensões limitadas dos painéis, em função do tamanho da prensa, não foi possível utilizar normas usualmente empregadas em trabalhos da literatura para esse produto. Adaptações foram feitas para as dimensões dos corpos de prova de flexão estática (410 mm x $50 \mathrm{~mm}$ ) e compressão paralela (120 mm x $50 \mathrm{~mm}$ ). Os ensaios mecânicos realizados foram flexão estática, compressão paralela às fibras e resistência ao cisalhamento na linha de cola nas condições seco, úmido e fervido. Os ensaios físicos consistiram das propriedades teor de umidade, absorção de água total e densidade aparente.

\subsection{Obtenção do MOE dinâmicos dos painéis com Stress Wave Timer}

Anteriormente ao ensaio destrutivo de flexão estática, realizaram-se as medições de propagação de onda com o uso do Stress Wave Timer da marca Metriguard nos mesmos corpos de prova. Foram realizadas medições paralelas e perpendiculares à direção das fibras da capa do painel, com dez medições em cada uma das direções.

$\mathrm{O}$ módulo de elasticidade dinâmico foi calculado em função da velocidade de propagação da onda, massa específica da lâmina e aceleração da gravidade, segundo a equação 1 :

$\operatorname{MOEd}=\delta \times V^{2} \times(1 / g)$

em que:

MOEd = módulo de elasticidade dinâmico $\left(\mathrm{kgf} / \mathrm{cm}^{2}\right)$; $\delta=$ massa específica da lâmina $\left(\mathrm{kg} / \mathrm{m}^{3}\right) ; \mathrm{g}=$ aceleração da gravidade $\left(9,804 \mathrm{~m} / \mathrm{s}^{2}\right) ; \mathrm{V}=$ velocidade da onda longitudinal $(\mathrm{cm} / \mathrm{s})$.

A velocidade é obtida pela Equação 2:

$\mathrm{V}=\mathrm{d} / \mathrm{t}$

em que:

$\mathrm{V}=$ velocidade $(\mathrm{cm} / \mathrm{s}) ; \mathrm{d}=$ distância entre os transdutores $(\mathrm{cm}) ; \mathrm{t}=$ tempo de propagação (s)

\subsection{Delineamento experimental e análise estatística dos dados}

Foi considerado um delineamento inteiramente casualizado com seis tratamentos e seis repetições,

Cerne, Lavras, v. 19, n. 3, p. 407-413, jul./set. 2013 
totalizando 36 painéis. Os resultados foram submetidos à análise de variância e as médias foram comparadas pelo teste de Scott-Knott a 5\% de significância.

O módulo de elasticidade dinâmico (MOEd) foi relacionado com o módulo de elasticidade estático (MOE) pelo modelo estatístico $\mathrm{y}=\mathrm{a}+\mathrm{b} / \mathrm{x}$, utilizando os dados médios de todas as repetições. A qualidade do modelo foi verificada pela análise de variância do modelo $(5 \%$ de significância), coeficiente de determinação e significância de seus coeficientes, avaliada pelo teste $t$. Todas as análises foram feitas a 5\% de significância.

\section{RESULTADOS E DISCUSSÃO}

\subsection{Propriedades físicas do painel LVL}

A umidade dos corpos-de-prova das espécies estudadas variou de $10,56 \%$ a $12,64 \%$. Na Tabela 3, são apresentados os resultados de densidade aparente e absorção de água total dos painéis.

Tabela 3 - Valores médios das propriedades físicas dos painéis LVL.

Table 3 - Average values of physical properties of LVL panels.

\begin{tabular}{ccc}
\hline Tratamento & $\begin{array}{c}\text { Densidade aparente } \\
\left(\mathrm{g} / \mathrm{cm}^{3}\right)\end{array}$ & $\begin{array}{c}\text { Absorção de Água Total } \\
(\%)\end{array}$ \\
\hline $\mathrm{Fr}$ & $0,669 \mathrm{a}$ & $36,2 \mathrm{~d}$ \\
$\mathrm{Ap}$ & $0,684 \mathrm{a}$ & $54,2 \mathrm{c}$ \\
$\mathrm{Fa}$ & $0,465 \mathrm{~d}$ & $69,4 \mathrm{~b}$ \\
$\mathrm{Pi}$ & $0,663 \mathrm{a}$ & $85,6 \mathrm{a}$ \\
$\mathrm{M}_{1}$ & $0,631 \mathrm{~b}$ & $56,8 \mathrm{c}$ \\
$\mathrm{M}_{2}$ & $0,586 \mathrm{c}$ & $72,5 \mathrm{~b}$ \\
\hline F calculado & $78,252 *$ & $45,099 *$ \\
$\mathrm{CV}(\%)$ & 3,58 & 10,03 \\
\hline
\end{tabular}

$\mathrm{e}^{*}$ : valores de F significativo a 5\% de significância. Médias seguidas pela mesma letra na coluna não diferem estatisticamente entre si pelo teste de Scott-Knott a 95\% de probabilidade.

Os painéis de freijó, amapá e Pinus oocarpa apresentaram valores de densidade aparente, estaticamente iguais e superiores em relação aos demais tratamentos. A menor densidade aparente foi observada para os painéis puros de faveira, o que resultou da baixa densidade básica dessa espécie.

Todos os painéis com inclusão das madeiras amazônicas tiveram absorção de água inferior aos painéis de Pinus oocarpa.

Cerne, Lavras, v. 19, n. 3, p. 407-413, jul./set. 2013
Para os painéis LVL, compostos por freijó e amapá, foi observada maior resistência à absorção de água em relação aos painéis compostos por faveira. De acordo com Tsoumis (1991), quanto maior a massa específica, menor é o teor de água que a madeira poderá conter na saturação. No entanto, os painéis de Pinus oocarpa apresentaram a maior absorção de água, mesmo com densidade aparente alta. Dessa forma, a qualidade dos painéis pode ser influenciada por outras propriedades além da densidade aparente. As madeiras de freijó e amapá apresentam ocorrência de tilos que obstruem os vasos impedindo a entrada de água nas lâminas de madeira (LIMA, 2011).

A presença de tilos também resultou em menores absorções de água para os painéis compostos por mistura de espécies, sendo as lâminas do freijó posicionadas na capa e as lâminas de amapá na transição. A inclusão de lâminas de Pinus oocarpa resultou em aumento da absorção de água, visto que menor absorção de água foi observada para os painéis da mistura 1 , em relação à mistura 2.

\subsection{Propriedades mecânicas dos painéis $L V L$}

Na Tabela 4, são apresentados os valores médios de compressão paralela e flexão estática.

Tabela 4 - Valores médios das propriedades mecânicas dos painéis LVL.

Table 4-Average values of mechanical properties of LVL panels.

\begin{tabular}{cccc}
\hline \multirow{2}{*}{ Tratamento } & \multirow{2}{*}{$\begin{array}{c}\text { Compressão } \\
\text { Paralela (MPa) }\end{array}$} & \multicolumn{2}{c}{ Flexão estática } \\
\cline { 3 - 4 } & $56,96 \mathrm{a}$ & $96,11 \mathrm{a}$ & $5.473,85 \mathrm{a}$ \\
$\mathrm{Fr}$ & $55,90 \mathrm{a}$ & $81,09 \mathrm{~b}$ & $5.145,30 \mathrm{a}$ \\
$\mathrm{Ap}$ & $33,70 \mathrm{c}$ & $65,80 \mathrm{~b}$ & $3.206,61 \mathrm{c}$ \\
$\mathrm{Fa}$ & $48,17 \mathrm{~b}$ & $74,49 \mathrm{~b}$ & $5.338,95 \mathrm{a}$ \\
$\mathrm{Pi}$ & $46,20 \mathrm{~b}$ & $94,12 \mathrm{a}$ & $5.382,35 \mathrm{a}$ \\
$\mathrm{M}_{1}$ & $43,63 \mathrm{~b}$ & $80,77 \mathrm{~b}$ & $4.739,88 \mathrm{~b}$ \\
$\mathrm{M}_{2}$ & $25,213^{*}$ & $3,314^{*}$ & $23,607^{*}$ \\
\hline F calculado & 8,2 & 18,9 & 8,8 \\
\hline CV (\%) & &
\end{tabular}

$\mathrm{e}^{*}$ : valor de F significativo a $5 \%$ de significância. Médias seguidas pela mesma letra na coluna não diferem estatisticamente entre si pelo teste de Scott-Knott a 95\% de probabilidade.

Para os painéis de freijó e amapá, foram observados valores de compressão paralela estatisticamente superiores aos obtidos para os painéis de Pinus oocarpa (testemunha) e demais tratamentos. Já, os painéis de faveira apresentaram 
valor significativamente menor para essa propriedade. Os valores de compressão paralela dos painéis compostos por mistura de lâminas foram significativamente iguais aos obtidos para os painéis de Pinus oocarpa. Guzmán e Hernández (2007) estudaram as propriedades de painéis LVL de Pinus radiata e encontraram valor médio de $32,45 \mathrm{MPa}$ para essa propriedade, inferior aos deste trabalho.

A norma NBR 7190 (ABNT, 1997) para estruturas de madeira estipula valor mínimo de compressão paralela de 38,48 MPa. Com exceção dos painéis de faveira, os valores obtidos para os painéis do presente trabalho são superiores ao mínimo reportado pela norma, o que atesta o bom desempenho mecânico desses produtos.

Considerando o módulo de ruptura e módulo de elasticidade em flexão estática, os painéis de freijó e da mistura 1 apresentaram valores superiores em relação aos obtidos para os painéis da testemunha e demais tratamentos. A maior densidade aparente do painel LVL de freijó certamente contribuiu para esse resultado. A variabilidade da maior parte das propriedades mecânicas e elásticas da madeira, segundo Panshin e Zeeuw (1980), pode ser estimada com base na variação da densidade. Dessa forma, os painéis de faveira apresentaram significativo menor módulo de elasticidade.

No caso da mistura 1, é importante mencionar que a capa foi composta por lâminas de freijó. Conforme mencionado por Iwakiri et al. (2008), as camadas externas do painel são as partes submetidas a maiores tensões de tração e compressão em flexão estática. No entanto, a inclusão da madeira de Pinus oocarpa, no painel composto pela mistura 2, ocasionou reduções nos valores de MOE e MOR. Nesse caso, é importante mencionar que madeiras de reflorestamento, normalmente são utilizadas quando ainda possuem madeira juvenil, a qual pode afetar o módulo de elasticidade, a tração paralela e perpendicular às fibras e a resistência à compressão paralela e normal de painéis laminados (KRETSCHMANN, 2008). Iwakiri et al. (2010) estudaram a influência da inclusão de madeira de Pinus oocarpa em painéis LVL produzidos com madeira de paricá e, também, observaram redução do módulo de elasticidade.

Na Tabela 5, são apresentados os valores médios de cisalhamento das amostras dos painéis.

Não houve efeito significativo da composição dos painéis sobre o cisalhamento nas condições seco, úmido e pós-fervura. Os valores encontrados neste trabalho para todos os painéis foram superiores aos apresentados por Iwakiri et al. (2010) para painéis LVL produzidos com
Tabela 5 - Valores médios de cisalhamento seco, úmido e pósfervido dos painéis LVL.

Table 5 - Average values of dry, humidity and boiled glue line shear strengh of LVL panels.

\begin{tabular}{cccc}
\hline \multirow{2}{*}{ Tratamento } & \multicolumn{3}{c}{ Cisalhamento (MPa) } \\
\cline { 2 - 4 } & Seco & Úmido & Pós-fervura \\
\hline $\mathrm{Fr}$ & $5,15 \mathrm{a}$ & $3,75 \mathrm{a}$ & $3,47 \mathrm{a}$ \\
$\mathrm{Ap}$ & $5,17 \mathrm{a}$ & $3,45 \mathrm{a}$ & $3,54 \mathrm{a}$ \\
$\mathrm{Fa}$ & $4,04 \mathrm{a}$ & $3,16 \mathrm{a}$ & $3,24 \mathrm{a}$ \\
$\mathrm{Pi}$ & $4,71 \mathrm{a}$ & $2,67 \mathrm{a}$ & $3,04 \mathrm{a}$ \\
$\mathrm{M}_{1}$ & $4,54 \mathrm{a}$ & $3,34 \mathrm{a}$ & $3,35 \mathrm{a}$ \\
$\mathrm{M}_{2}$ & $4,70 \mathrm{a}$ & $3,57 \mathrm{a}$ & $3,23 \mathrm{a}$ \\
\hline F calculado & $1,226^{\mathrm{ns}}$ & $1,456^{\mathrm{ns}}$ & $0,384^{\mathrm{ns}}$ \\
$\mathrm{CV}(\%)$ & 19,72 & 22,95 & 21,78 \\
\hline
\end{tabular}

$\mathrm{e}^{\text {ns: }}$ valor de F não significativo a $5 \%$ de significância Médias seguidas pela mesma letra na coluna não diferem estatisticamente entre si pelo teste de Scott-Knott a 95\% de probabilidade.

a madeira da espécie amazônica paricá. No entanto, os autores utilizaram adesivo fenol-formaldeído, sendo uma maior resistência observada para o adesivo resorcinolformaldeído.

\subsection{Obtenção do MOE dinâmico dos painéis com Stress Wave Timer}

$\mathrm{Na}$ Figura 2, apresenta-se, graficamente, a correlação entre MOE estático e MOE dinâmico e a dispersão dos valores observados.

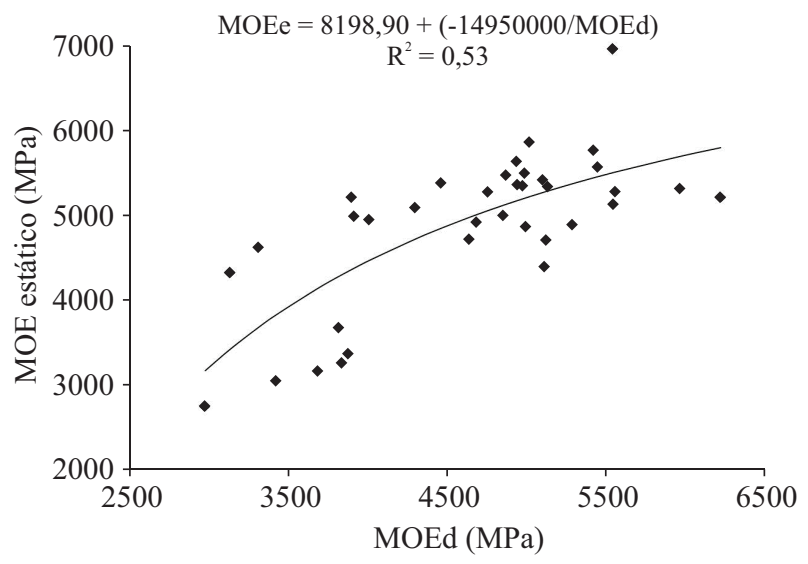

Figura 2 - Gráfico de correlação entre o MOE estático e o MOE dinâmico.

Figure 2 - Correlation graphic between static and dynamic $M O E$.

Cerne, Lavras, v. 19, n. 3, p. 407-413, jul./set. 2013 
O modelo de regressão ajustado foi significativo (Fcalculado $=38,59$; p-valor $<0,05 \%$ ), assim como os coeficientes a (tcalculado $=15,073$; $\mathrm{p}$-valor $<0,05 \%$ ) e $\mathrm{b}$ (tcalculado $=-6,213 ; \mathrm{p}$-valor $<0,05 \%)$. O coeficiente de determinação encontrado indica que $53 \%$ da variação do módulo de elasticidade estático ocorre em função da variabilidade do módulo de elasticidade dinâmico, sendo que os outros $47 \%$ possam ser explicados por variáveis como matéria-prima, as condições de manufatura dos painéis entre outras.

Avaliando painéis LVL produzidos com Pinus Kesiya para flanges de vigas em "I", Santos (2008) observou que a relação existente entre o módulo de elasticidade dinâmico e estático apresentou um coeficiente de determinação de 0,5197. Gabriel (2007) verificou as relações existentes entre módulo de elasticidade dinâmico e estático em painéis de Pinus caribeae (var. caribeae, bahamensis e hondurensis) e Pinus oocarpa e observou alto coeficiente de determinação de 0,696.

\section{CONCLUSÕES}

Todos os painéis LVL produzidos com espécies amazônicas apresentaram maior resistência á absorção de água em relação aos painéis produzidos com a espécie comercial Pinus oocarpa. Dentre as espécies amazônicas, os painéis de faveira apresentaram maior absorção de água, o que foi atribuído, principalmente, à baixa densidade básica dessa espécie que resulta em baixa densidade aparente do painel.

Para compressão paralela, os painéis de freijó e amapá apresentaram melhor desempenho. Os painéis compostos por freijó e a mistura 1 apresentaram maior módulo de ruptura. Já, para o módulo de elasticidade, os painéis de faveira e a mistura 2 apresentaram menor resistência, enquanto que para os demais não foi observada diferença significativa.

Para resistência à colagem, não houve diferença significativa entre os painéis.

O stress wave timer pode ser utilizado para predição do MOE estático, a partir do MOE dinâmico.

\section{REFERÊNCIAS}

ARAÚJO, H. J. B. Agrupamentos das espécies madeireiras ocorrentes em pequenas áreas sob manejo florestal do Projeto de colonização Pedro Peixoto (AC) por similaridade das propriedades físicas e mecânicas. 2002. 184 p. Dissertação (Mestrado em Recursos Florestais) - Escola Superior de Agricultura “Luiz de Queiroz”, Piracicaba, 2002.
ASSOCIAÇÃO BRASILEIRA DE NORMAS TÉCNICAS. ABNT/CB-31: projeto de normas 31.000.05.001: chapas de madeira compensada. Rio de Janeiro, 2004.

ASSOCIAÇÃO BRASILEIRA DE NORMAS TÉCNICAS. NBR 7190: projeto de estruturas de madeira. Rio de Janeiro, 1997. $107 \mathrm{p}$.

BARBOSA, A. P.; VIANEZ, B. F.; VAREJÃO, M. de J.; ABREU, R. S. de. Consideração sobre o perfil tecnológico do setor madeireiro na Amazônia Central. Biodiversidade, Pesquisa e Desenvolvimento na Amazônia, Manaus, n. 12, p. 421, 2001.

BORTOLETTO JÚNIOR, G. Estudo comparativo das propriedades físicas e mecânicas da madeira e do LVL de Pinus merkusii. Revista Forestal Venezolana, Merida, v. 53, n. 2, p. 191-195, 2009.

BURDURLU, E.; KILIC, M.; ILCE, A. C.; UZUNKAVAK, $\mathrm{O}$. The effects of ply organization and direction on bending strength and modulus of elasticity in laminated veneer lumber (LVL) obtained from beech (Fagus orientalis L.) and lombardy poplar (Populus nigra L.). Construction and Building Materials, Ankara, v. 21, n. 8, p. 1720-1725, 2007.

CARVALHO, A. M.; LAHR, F. A. R.; BOTOLETTO JÚNIOR, G. Use of Brazilian eucalyptus to produce LVL panels. Forest Products Journal, Madison, v. 54, n. 11, p. 61-64, 2004.

COLAK, S.; COLAKOGLU, G.; AYDIN, I. Effects of log steaming, veneer drying and aging on the mechanical properties of laminated veneer lumber (LVL). Building and Environment, West Lafayette, v. 42, n. 1, p. 93-98, 2007.

DING, W. E.; ABDUL-KADER, R.; KAWAI, S. Properties of Rubberwood LVL reinforced with Acacia Veneers. In: WORLD CONGRESS, 20., 1995, Finland. Proceedings... Finland: IUFRO, 1995. p. 8-16.

GABRIEL, M. S. C. Desempenho físico-mecânico de painéis LVLs de Pinus tropicais da região de São Paulo. 2007. 125

f. Tese (Doutorado em Energia na Agricultura) - Universidade Estadual Paulista, Botucatu, 2007.

GUZMÁN, F. M.; HERNÁNDEZ, C. G. Propriedades físicas y mecánicas. In: _. LVL vigas de chapas laminadas confeccionadas com Pinus radiata y Eucalyptus nitens. Concepción: INFOR, 2007. p. 12-32.

Cerne, Lavras, v. 19, n. 3, p. 407-413, jul./set. 2013 
H'NG, P. S.; PARIDAH, M. T.; CHIN, K. L. Bending properties of laminated veneer lumber produced from keruing (dipterocarpus sp.) reinforced with low density wood species. Asian Journal of Scientific Research, Malaysia, v. 3, n. 2, p. $1-8,2010$.

IWAKIRI, S.; MATOS, J. L. M. de; PINTO, J. A.; VIANA, L. C.; SOUZA, M. M. de; TRIANOSKI, R.; ALMEIDA, V. C. Produção de painéis laminados unidirecionais-LVL com lâminas de Schizolobium amazonicum, Eucalyptus saligna e Pinus taeda. Cerne, Lavras, v. 16, n. 4, p. 557-563, 2010.

IWAKIRI, S.; MATOS, J. L. M. de; PRATA, J. G.; TORQUATO, L. P.; BRONOSKI, M.; NISHIDATE, M. M. Produção de painéis laminados unidirecional: LVL com madeiras de Eucalyptus grandis Hill ex Maiden e Eucalyptus dunnii Maiden. Floresta e Ambiente, Seropédica, v. 15, n. 2 , p. 1-7, 2008.

KRETSCHMANN, D. E. The influence of juvenile Wood content on shear parallel, compression, and tension perpendicular to grain strength and mode fracture toughness of loblolly pine at various ring orientation. Forest Productions Journal, Madison v. 58, n. 7/8, p. 8996, 2008.

LIMA, N. N. Laminados e particulados à base de madeiras tropicais da Amazônia. 2011. 207 p. Tese (Doutorado em Ciência e Tecnologia da Madeira) - Universidade Federal de Lavras, Lavras, 2011.
MCKEEVER, D. B. Engineered Wood products: a response to the changing timber resource. Pacific Rim Wood Market Report, Gig Harbor, n. 123, p. 5-15, 1997.

MOHAMAD, W. H. W.; RAZLAN, M. A.; AHMAD, Z. Bending strength properties of glued laminated timber from selected Malaysian hardwood timber. International Journal of Civil \& Environmental Engineering, Padova, v. 11, n. 4, p. 7-12, 2011.

MOODY, R. C.; HERNANDEZ, R. Glued-laminated timber. In: Engineered wood products-A guide for

specifiers, designers and users. Madison: USDA Forest Service, 1997. p. 1-39.

ÖZÇIFÇI, A. Effects of scarf joints on bending strength and modulus of elasticity to laminated veneer lumber (LVL). Building and Environment, West Lafayette, v. 42, n. 3, p. 1510-1514, 2007.

PANSHIN, A. J.; ZEEUW, C. de. Textbook of wood technology. New York: McGraw-Hill, 1980. 722 p.

SANTOS, A. M. L. Avaliação teórica e experimental de vigas em "I" pré-fabricadas de madeira com flange de Painéis de Lâminas Paralelas (LVL) e Alma de Painéis de Partículas Orientadas (OSB) e Compensado. 2008. 94 p. Dissertação (Mestrado em Ciências Florestais) - Universidade de Brasília, Brasília, 2008.

TSOUMIS, G. Science and technology of wood: structure, properties, utilization. New York: Chapman \& Hall, 1991.

Recebido: 4 de maio de 2011; aceito: 25 de janeiro de 2013.

Cerne, Lavras, v. 19, n. 3, p. 407-413, jul./set. 2013 
